\title{
Irreducible tensors and selection rules for three-frequency absorption
}

David L. Andrews and Philip J. Wilkes ${ }^{\text {a) }}$

School of Chemical Sciences, University of East Anglia, Norwich NR4 7TJ, England

(Received 10 October 1984; accepted 29 March 1985)

In this paper rate equations and selection rules are derived for the most general case of threephoton absorption, where the photons all have different frequencies and directions of propagation. The rate equations are re-expressed in terms of irreducible tensor products, and the relationship between these and the more familiar isotropic molecular parameters is given in full for the first time. The selection rules are examined in detail and a classification scheme for the allowed transitions is given together with a method of experimentally distinguishing between the classes. It is shown that once assignment of a transition to one of the 11 possible classes has been carried out, the symmetry of the excited state can in many cases be unambiguously identified. The connection with the classification schemes previously developed for single and double-beam three-photon absorption is also discussed, and finally, a special experimental arrangement is proposed which should give rise to a spectrum in which the only transitions to occur are those forbidden by both single and double-beam excitation.

\section{INTRODUCTION}

Progress in multiphoton spectroscopy is increasingly drawing attention to its unique potential for characterizing molecular excited states. The state of development of laser technology is now such that two-photon spectroscopy has become a well-established method, and three-photon studies are now being looked at with fresh interest.

In this paper we develop the theory of three-beam threephoton absorption, following previous studies which have dealt with the other two cases of three-photon absorption involving single-beam and double-beam excitation. Such studies should be practicable using three frequencies derived from a single pulsed laser source by means of frequency conversion, as for example in a tunable dye laser.

The selection rules for three-frequency excitation differ somewhat from those applicable to other three-photon absorption studies, and thereby provide access to certain states which would otherwise be inaccessible. More important is the fact that much fuller symmetry information on threephoton allowed states is provided by a three-frequency study. We demonstrate that it should be experimentally possible to assign each band in a three-frequency spectrum to one of 11 classes, which generally results in an unambiguous symmetry assignment for each corresponding excited state.

\section{IRREDUCIBLE TENSOR FORMULATION OF RATE EQUATIONS}

The rate of three-photon absorption, calculated by standard quantum electrodynamical methods using timedependent perturbation theory is as follows:

$$
\Gamma=K\left\langle\left|T_{i j k} \mathbf{e}_{1 i} \mathbf{e}_{2 j} \mathbf{e}_{3 k}\right|^{2}\right\rangle,
$$

where the factor $K$ is given by

$$
K=\frac{16 \pi^{4}}{\hbar c^{3}} I_{1} I_{2} I_{3} \rho_{f}
$$

Here $I_{n}$ and $\mathbf{e}_{n}$ represent the irradiance and polarization vector of beam $n$ with frequency $\omega_{n}$ and the angular brackets as usual represent the rotational averaging which is required in the development of rate equations applicable to fluid media. For reasons which will become evident later, we stipulate that all three beams have different frequencies.

The molecular response tensor $T_{i j k}$ in Eq. (2.1) is given by

$$
\begin{aligned}
T_{i j k}= & \sum_{r, s}\left[\frac{\mu_{k}^{f s} \mu_{j}^{s r} \mu_{i}^{r 0}}{\left(\widetilde{E}_{s 0}-\hbar \omega_{1}-\hbar \omega_{2}\right)\left(\widetilde{E}_{r 0}-\hbar \omega_{1}\right)}+\frac{\mu_{j}^{f s} \mu_{k}^{s r} \mu_{i}^{\circ 0}}{\left(\widetilde{E}_{s 0}-\hbar \omega_{1}-\hbar \omega_{3}\right)\left(\widetilde{E}_{r 0}-\hbar \omega_{1}\right)}\right. \\
& +\frac{\mu_{k}^{f s} \mu_{i}^{s r} \mu_{j}^{\circ 0}}{\left(\widetilde{E}_{s 0}-\hbar \omega_{1}-\hbar \omega_{2}\right)\left(\widetilde{E}_{r 0}-\hbar \omega_{2}\right)}+\frac{\mu_{i}^{f s} \mu_{k}^{s r} \mu_{j}^{\circ 0}}{\left(\widetilde{E}_{s 0}-\hbar \omega_{2}-\hbar \omega_{3}\right)\left(\widetilde{E}_{r 0}-\hbar \omega_{2}\right)} \\
& \left.+\frac{\mu_{j}^{f s} \mu_{i}^{s r} \mu_{k}^{r 0}}{\left(\widetilde{E}_{s 0}-\hbar \omega_{1}-\hbar \omega_{3}\right)\left(\widetilde{E}_{r 0}-\hbar \omega_{3}\right)}+\frac{\mu_{i}^{f s} \mu_{j}^{s r} \mu_{k}^{\circ 0}}{\left(\widetilde{E}_{s 0}-\hbar \omega_{2}-\hbar \omega_{3}\right)\left(\widetilde{E}_{r 0}-\hbar \omega_{3}\right)}\right],
\end{aligned}
$$

where $\widetilde{E}_{x 0}$ and $\widetilde{E}_{s 0}$ are complex energy terms incorporating damping factors to model resonance line shapes, i.e., $\widetilde{E}_{\kappa}$

\footnotetext{
a) Present address: Department of Chemistry, Science Laboratories, University of Durham, South Road, Durham DH1 3LE, England.
}

$=E_{s 0}-\frac{1}{2} i \hbar \gamma_{r}$ and $\widetilde{E}_{s 0}=E_{s 0}-\frac{1}{2} i \hbar \gamma_{s}$. When the frequencies of irradiation are far away from resonance with any molecular energy levels, these damping factors may be ignored, and the tensor becomes real. However for generality we shall assume that the tensor is complex in the following calculations. 
TABLE I. Linearly independent basis sets of polarization parameters $a_{n}$ and isotropic molecular parameters $t_{n}$

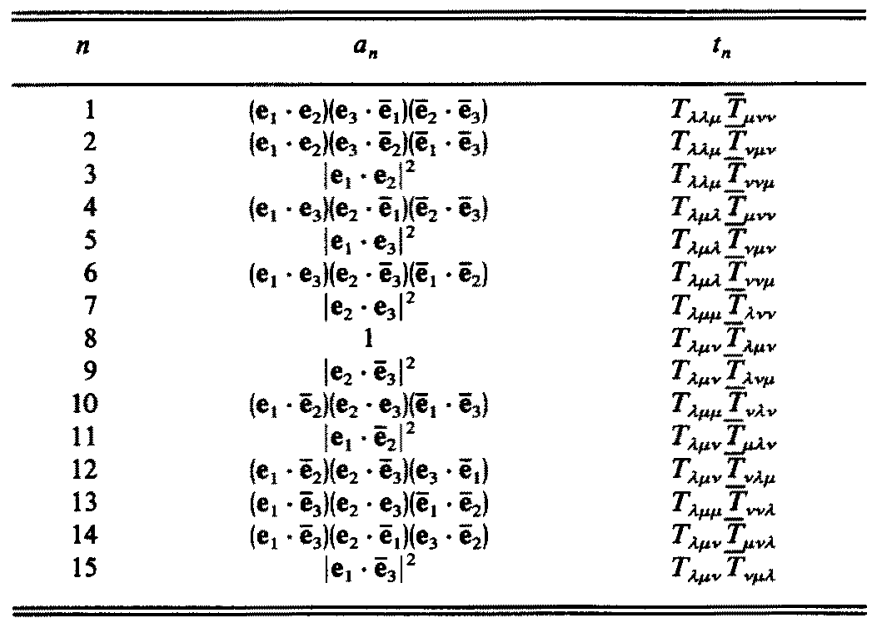

The rotational average in Eq. (2.1) may be written as

$$
\begin{aligned}
\left\langle\left|T_{i j k} \mathbf{e}_{1 i} \mathbf{e}_{2 j} \mathbf{e}_{3 k}\right|^{2}\right\rangle= & \left\langle T_{i j k} \bar{T}_{l m n} \mathbf{e}_{1 i} \mathbf{e}_{2 j} \mathbf{e}_{3 k} \overline{\mathbf{e}}_{1 l} \overline{\mathbf{e}}_{2 m} \overline{\mathbf{e}}_{3 n}\right\rangle \\
= & T_{\lambda \mu \nu} \bar{T}_{\text {omp }} \mathbf{e}_{1 i} \mathbf{e}_{2 j} \mathbf{e}_{3 k} \overline{\mathbf{e}}_{1 i} \overline{\mathbf{e}}_{2 m} \overline{\mathbf{e}}_{3 n} \\
& \times I_{i j k l m n ;}^{(6)} \lambda \mu v o \pi \rho
\end{aligned}
$$

where $I^{(6)}$ is the sixth rank rotational average, as given in Ref. 1 , and the Greek indices denote a molecular coordinate system to which components of the molecular tensor are now referred. The formula for $I^{(6)}$ is as follows:

$$
I_{i j k l m n ; \lambda \mu v o \pi \rho}^{(6)}=\sum_{p, q}^{15} m_{p q} f_{i j k l m n}^{(6 ; p)} g_{\lambda \mu v o \pi \rho}^{(6 ; q)},
$$

where $m_{p q}$ are numerical coefficients and $f^{(6 ; p)}$ and $g^{(6 ; 9)} \mathrm{de}-$ note triple products of Kronecker deltas such as $\delta_{i j} \delta_{k l} \delta_{m n}$ and $\delta_{\lambda \mu} \delta_{v o} \delta_{\pi \rho}$. From Eqs. (2.1), (2.4), and (2.5) we thus obtain a rate given by

$$
\Gamma=K \sum_{p, q}^{15} a_{p} m_{p q}^{(6)} t_{q}
$$

where the $a_{p}$ form a set of 15 parameters determined by polarization conditions, and $t_{q}$ a corresponding set of isotropic molecular parameters as given in Table I (see also Ref. 2).

Since the selection rules associated with each of the molecular parameters $t_{q}$ are not generally clear cut, it is preferable to re-express the result in terms of irreducible tensors. The molecular response tensor may be expressed in terms of irreducible parts of weights 0 to 3 :

$$
\begin{aligned}
T_{\lambda \mu \nu}= & T_{\lambda \mu \nu}^{(0)}+T_{\lambda \mu \nu}^{(1 \alpha)}+T_{\lambda \mu \nu}^{(1 \beta)}+T_{\lambda \mu \nu}^{(1 \mu)} \\
& +T_{\lambda \mu \nu}^{(2 \alpha)}+T_{\lambda \mu \nu}^{(2 \beta)}+T_{\lambda \mu \nu}^{(3)} .
\end{aligned}
$$

\begin{tabular}{|c|c|}
\hline$n$ & $t_{n}^{i}$ \\
\hline 1 & $T_{\lambda \mu \nu}^{(0)} \bar{T}_{\lambda \mu \nu}^{(0)}$ \\
\hline 2 & $T_{\lambda \mu \nu}^{(\alpha)} \bar{T}_{\lambda \mu \nu}^{(1 \alpha)}$ \\
\hline 3 & $T_{\lambda \mu \nu}^{(1 \alpha)} \bar{T}_{\lambda \mu \nu}^{(1 \beta)}$ \\
\hline 4 & 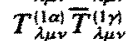 \\
\hline 5 & $T_{\lambda \mu \nu}^{(1 \beta)} \bar{T}_{\lambda \mu \nu}^{(1 \alpha)}$ \\
\hline 6 & $T_{\lambda \mu \nu}^{(1 \beta)} \bar{T}_{\lambda \mu \nu}^{(1 \beta)}$ \\
\hline 7 & $T_{\lambda \mu \nu}^{(\beta)} \bar{T}_{\lambda \mu \nu}^{(1) \gamma}$ \\
\hline 8 & $T_{\lambda \mu v}^{(1) \gamma} \bar{T}_{\lambda \mu \nu}^{(1 \alpha)}$ \\
\hline 9 & $T_{\lambda \mu \nu}^{(1)} \bar{T}_{\lambda \mu \nu}^{(1 \beta)}$ \\
\hline 10 & 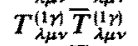 \\
\hline 11 & $T_{\lambda \mu \nu}^{(2 \alpha)} \bar{T}_{\lambda \mu \nu}^{(2 \alpha)}$ \\
\hline 12 & $T_{\lambda \mu \nu}^{(2 \alpha)} \bar{T}_{\lambda \mu \nu}^{(2 \beta)}$ \\
\hline 13 & $T_{\lambda \mu \nu}^{(2 \beta)} \bar{T}_{\lambda \mu \nu}^{(2 \alpha)}$ \\
\hline 14 & $T_{\lambda \mu \nu}^{(2 \beta)} \bar{T}_{\lambda \mu \nu}^{(2 \beta)}$ \\
\hline 15 & $T_{\lambda \mu \nu}^{(3)} \bar{T}_{\lambda \mu \nu}^{(3)}$ \\
\hline
\end{tabular}

Explicit expressions for the irreducible tensors in (2.7) are as follows:
TABLE II. Linearly independent basis set of molecular irreducible tensor products.

$$
\begin{aligned}
& T_{\lambda \mu v}^{(0)}=\frac{1}{6} \epsilon_{\lambda \mu \nu} \epsilon_{\rho \sigma \tau} T_{\rho \sigma \tau}, \\
& T_{\lambda \mu \nu}^{(1 \alpha)}=\frac{1}{10}\left(4 \delta_{\lambda \mu} T_{\rho \rho v}-\delta_{\lambda v} T_{\rho \rho \mu}-\delta_{\mu v} T_{\rho \rho \lambda}\right), \\
& T_{\lambda \mu \nu}^{(1 \beta)}=110\left(-\delta_{\lambda \mu} T_{\rho v \rho}+4 \delta_{\lambda v} T_{\rho \mu \rho}-\delta_{\mu v} T_{\rho \lambda \rho}\right) \text {, } \\
& T_{\lambda \mu \nu}^{(1)}=\frac{1}{10}\left(-\delta_{\lambda \mu} T_{\nu \rho \rho}-\delta_{\lambda \nu} T_{\mu \rho \rho}+4 \delta_{\mu \nu} T_{\lambda \rho \rho}\right) \\
& T_{\lambda \mu \nu}^{(2 \alpha)}=\frac{1}{6} \epsilon_{\lambda \mu \tau}\left(2 \epsilon_{\rho \sigma \tau} T_{\rho \sigma \tau}+2 \epsilon_{\rho \sigma v} T_{\rho \sigma \tau}+\epsilon_{\rho \sigma \tau} T_{v \rho \sigma}\right. \\
& \left.+\epsilon_{\rho \sigma v} T_{\tau \rho \sigma}-2 \delta_{v \tau} \epsilon_{\pi \rho \sigma} T_{\pi \rho \sigma}\right) \\
& T_{\lambda \mu \nu}^{(2 \beta)}=\frac{1}{6} \epsilon_{\mu v \tau}\left(2 \epsilon_{\rho \sigma \tau} T_{\lambda \rho \sigma}+2 \epsilon_{\rho \sigma \lambda} T_{\tau \rho \sigma}+\epsilon_{\rho \sigma \tau} T_{\rho \sigma \lambda}\right. \\
& \left.+\epsilon_{\rho \sigma \lambda} T_{\rho \sigma \tau}-2 \delta_{\lambda \tau} \epsilon_{\pi \rho \sigma} T_{n \rho \sigma}\right) \\
& T_{\lambda \mu \nu}^{(3)}=\frac{1}{6}\left(T_{\lambda \mu \nu}+T_{\lambda \nu \mu}+T_{\mu \lambda \nu}+T_{\mu \nu \lambda}+T_{\nu \lambda \mu}+T_{\nu \mu \lambda}\right) \\
& -\frac{1}{15}\left[\delta_{\lambda \mu}\left(T_{\rho \rho v}+T_{\rho v \rho}+T_{\nu \rho \rho}\right)+\delta_{\lambda v}\left(T_{\rho \rho \mu}\right.\right. \\
& \left.\left.+T_{\rho \mu \rho}+T_{\mu \rho \rho}\right)+\delta_{\mu v}\left(T_{\rho \rho \lambda}+T_{\rho \lambda \rho}+T_{\lambda \rho \rho}\right)\right]
\end{aligned}
$$

The formulas for the tensor reduction are taken from Ref. 3 . In passing, we note that in previous applications to threephoton processes where two photons are identical, $T_{\lambda \mu \nu}^{(1 \alpha)}$ and $T_{\lambda \mu \nu}^{(\beta)}$ are not independent, and their sum has been represented by $T_{\lambda \mu \nu}^{(1 A)}$, while $T_{\lambda \mu \nu}^{(1)}$ has been written as $T_{\lambda \mu \nu}^{(1 B)}$. Similarly the sum of $T_{\lambda \mu \nu}^{(2 \alpha)}$ and $T_{\lambda \mu \nu}^{(2 \beta)}$ has simply been represented by $T_{\lambda \mu \nu}^{(2)}$ in such cases.

The irreducible tensors have the following transformation properties under the operations of the full rotation group:

(i) $T_{\lambda \mu \nu}^{(0)}$ is fully antisymmetric in all its indices and it transforms as a pseudoscalar.

(ii) $T_{\lambda \mu \nu}^{(1 \alpha)}, \quad T_{\lambda \mu \nu}^{(1 \beta)}$, and $T_{\lambda \mu \nu}^{(1 \gamma)}$ transform as polar vectors.

(iii) $T_{\lambda \mu \nu}^{(2 \alpha)}$ and $T_{\lambda \mu \nu}^{(2 \beta)}$ transform as traceless, index-symmetric second rank tensors.

(iv) $T_{\lambda \mu v}^{(3)}$ transforms as a traceless, fully index-symmetric third rank polar tensor.

Each irreducible tensor of weight $n$ has $(2 n+1)$ independent components hence giving $T_{\lambda \mu \nu} 27$ components (as required by a third rank tensor).

It is worth noting that $T_{\lambda \mu \nu}^{(0)}$, the particular significance of which will shortly become evident, is zero if two or more 
TABLE III. Matrix of coefficients relating the irreducible tensor products to the isotropic molecular parameters.

\begin{tabular}{|c|c|c|c|c|c|c|c|c|c|c|c|c|c|c|c|}
\hline & 0 & 0 & 0 & 0 & 0 & 0 & 0 & 15 & -15 & 0 & -15 & 15 & 0 & 15 & -15 \\
\hline \multirow{14}{*}{$\frac{1}{90}$} & 0 & 0 & 36 & 0 & 0 & 0 & 0 & 0 & 0 & 0 & 0 & 0 & 0 & 0 & 0 \\
\hline & 0 & -9 & 0 & 0 & 0 & 0 & 0 & 0 & 0 & 0 & 0 & 0 & 0 & 0 & 0 \\
\hline & -9 & 0 & 0 & 0 & 0 & 0 & 0 & 0 & 0 & 0 & 0 & 0 & 0 & 0 & 0 \\
\hline & 0 & 0 & 0 & 0 & 0 & -9 & 0 & 0 & 0 & 0 & 0 & 0 & 0 & 0 & 0 \\
\hline & 0 & 0 & 0 & 0 & 36 & 0 & 0 & 0 & 0 & 0 & 0 & 0 & 0 & 0 & 0 \\
\hline & 0 & 0 & 0 & -9 & 0 & 0 & 0 & 0 & 0 & 0 & 0 & 0 & 0 & 0 & 0 \\
\hline & 0 & 0 & 0 & 0 & 0 & 0 & 0 & 0 & 0 & 0 & 0 & 0 & -9 & 0 & 0 \\
\hline & 0 & 0 & 0 & 0 & 0 & 0 & 0 & 0 & 0 & -9 & 0 & 0 & 0 & 0 & 0 \\
\hline & 0 & 0 & 0 & 0 & 0 & 0 & 36 & 0 & 0 & 0 & 0 & 0 & 0 & 0 & 0 \\
\hline & 20 & -10 & -10 & 20 & -10 & -10 & -40 & 40 & 40 & 20 & -20 & -20 & 20 & -20 & -20 \\
\hline & -5 & -5 & 10 & -5 & -5 & 10 & 10 & -10 & -10 & 10 & -10 & -10 & -20 & 20 & 20 \\
\hline & -20 & 10 & 10 & 10 & -5 & -5 & 10 & -10 & -10 & -5 & -10 & 20 & -5 & -10 & 20 \\
\hline & 20 & 20 & -40 & -10 & -10 & 20 & -10 & 40 & -20 & -10 & 40 & -20 & 20 & -20 & -20 \\
\hline & -6 & -6 & -6 & -6 & -6 & -6 & -6 & 15 & 15 & -6 & 15 & 15 & -6 & 15 & 15 \\
\hline
\end{tabular}

of the excitation frequencies are identical; hence we stipulate that $\omega_{1} \neq \omega_{2} \neq \omega_{3} \neq \omega_{1}$.

The molecular parameters $t_{q}$ in Eq. (2.6) are all tensor products, e.g., $T_{\lambda \lambda \mu} \bar{T}_{\mu \nu \nu}$ and as indicated by Eq. (2.7) such products can be re-expressed in terms of irreducible tensor products $t_{r}{ }_{r}$. Since the inner product of tensors with unequal weights is zero, owing to the index symmetry properties, there are fifteen such products, as listed in Table II. For simplicity, these are all expressed in the form $T_{\lambda \mu \nu}^{(\omega)} \bar{T}_{\lambda \mu \nu}^{\left(w^{\prime}\right)}{ }^{4}$

The $15 t_{r}^{i}$ parameters can be evaluated using the Eq. (2.8) and expressed in terms of the $t_{q}$. The relationship between $t_{r}^{i}$ and $t_{q}$ takes the form:

$$
t_{r}^{i}=\sum_{q}^{15} j_{r q} t_{q} .
$$

The matrix of coefficients $j_{r q}$ for this equation is given in Table III.

In order to obtain the rate expression (2.6) in terms of the irreducible tensor products $t_{r}^{i}$ it is necessary to find the inverse of Eq. (2.9), i.e.,

$$
t_{q}=\sum_{r}^{15}\left(j^{-1}\right)_{q r} t_{r}^{i}
$$

The result obtained is as follows:

$$
\left[\begin{array}{l}
T_{\lambda \mu \mu} \bar{T}_{\mu \nu \nu} \\
T_{\lambda \mu \mu} \bar{T}_{v \mu \nu} \\
T_{\lambda \lambda \mu} \bar{T}_{\nu \nu \mu} \\
T_{\lambda \mu \lambda} \bar{T}_{\mu v \nu} \\
T_{\lambda \mu \lambda} \bar{T}_{v \mu \nu} \\
T_{\lambda \mu \lambda} \bar{T}_{v v \mu} \\
T_{\lambda \mu \mu} \bar{T}_{\lambda \nu \nu} \\
T_{\lambda \mu \nu} \bar{T}_{\lambda \mu \nu} \\
T_{\lambda \mu \nu} \bar{T}_{\lambda \nu \mu} \\
T_{\lambda \mu \mu} \bar{T}_{v \lambda \nu} \\
T_{\lambda \mu \nu} \bar{T}_{\mu \lambda \nu} \\
T_{\lambda \mu \nu \nu} \bar{T}_{\nu \lambda \mu} \\
T_{\lambda \mu \mu} \bar{T}_{v \nu \lambda} \\
T_{\lambda \mu \nu} \bar{T}_{\mu \nu \lambda} \\
T_{\lambda \mu \nu} \bar{T}_{v \mu \lambda}
\end{array}\right]
$$

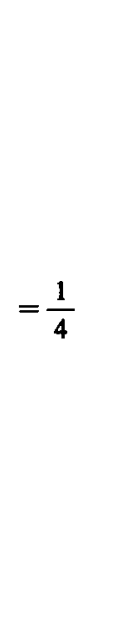

$$
\left[\begin{array}{rrrrrrrrrrrrrrr}
0 & 0 & 0 & -40 & 0 & 0 & 0 & 0 & 0 & 0 & 0 & 0 & 0 & 0 & 0 \\
0 & 0 & -40 & 0 & 0 & 0 & 0 & 0 & 0 & 0 & 0 & 0 & 0 & 0 & 0 \\
0 & 10 & 0 & 0 & 0 & 0 & 0 & 0 & 0 & 0 & 0 & 0 & 0 & 0 & 0 \\
0 & 0 & 0 & 0 & 0 & 0 & -40 & 0 & 0 & 0 & 0 & 0 & 0 & 0 & 0 \\
0 & 0 & 0 & 0 & 0 & 10 & 0 & 0 & 0 & 0 & 0 & 0 & 0 & 0 & 0 \\
0 & 0 & 0 & 0 & -40 & 0 & 0 & 0 & 0 & 0 & 0 & 0 & 0 & 0 & 0 \\
0 & 0 & 0 & 0 & 0 & 0 & 0 & 0 & 0 & 10 & 0 & 0 & 0 & 0 & 0 \\
4 & 4 & 4 & 4 & 4 & 4 & 4 & 4 & 4 & 4 & 4 & 4 & 4 & 4 & 4 \\
-4 & -1 & -16 & 4 & -16 & -1 & 4 & 4 & 4 & 4 & 2 & -4 & -4 & -4 & 4 \\
0 & 0 & 0 & 0 & 0 & 0 & 0 & 0-40 & 0 & 0 & 0 & 0 & 0 & 0 \\
-4 & 4 & 4 & 4 & 4 & -1-16 & 4-16 & -1 & -4 & -4 & -4 & 2 & 4 \\
4 & -1 & 4 & -16 & -16 & -1 & 4 & 4-16 & -1 & -2 & -8 & 4 & -2 & 4 \\
0 & 0 & 0 & 0 & 0 & 0 & 0 & -40 & 0 & 0 & 0 & 0 & 0 & 0 & 0 \\
4 & -1 & -16 & 4 & 4 & -1 & -16 & -16 & 4 & -1 & -2 & 4 & -8 & -2 & 4 \\
-4 & -1 & 4 & -16 & 4 & 4 & 4-16 & 4 & -1 & 2 & 8 & 8 & 2 & 4
\end{array}\right]
$$

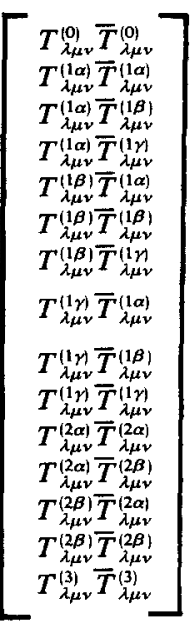

Substituting the result (2.11) into Eq. (2.6) gives the following expression for the rate in terms of the irreducible tensors: 


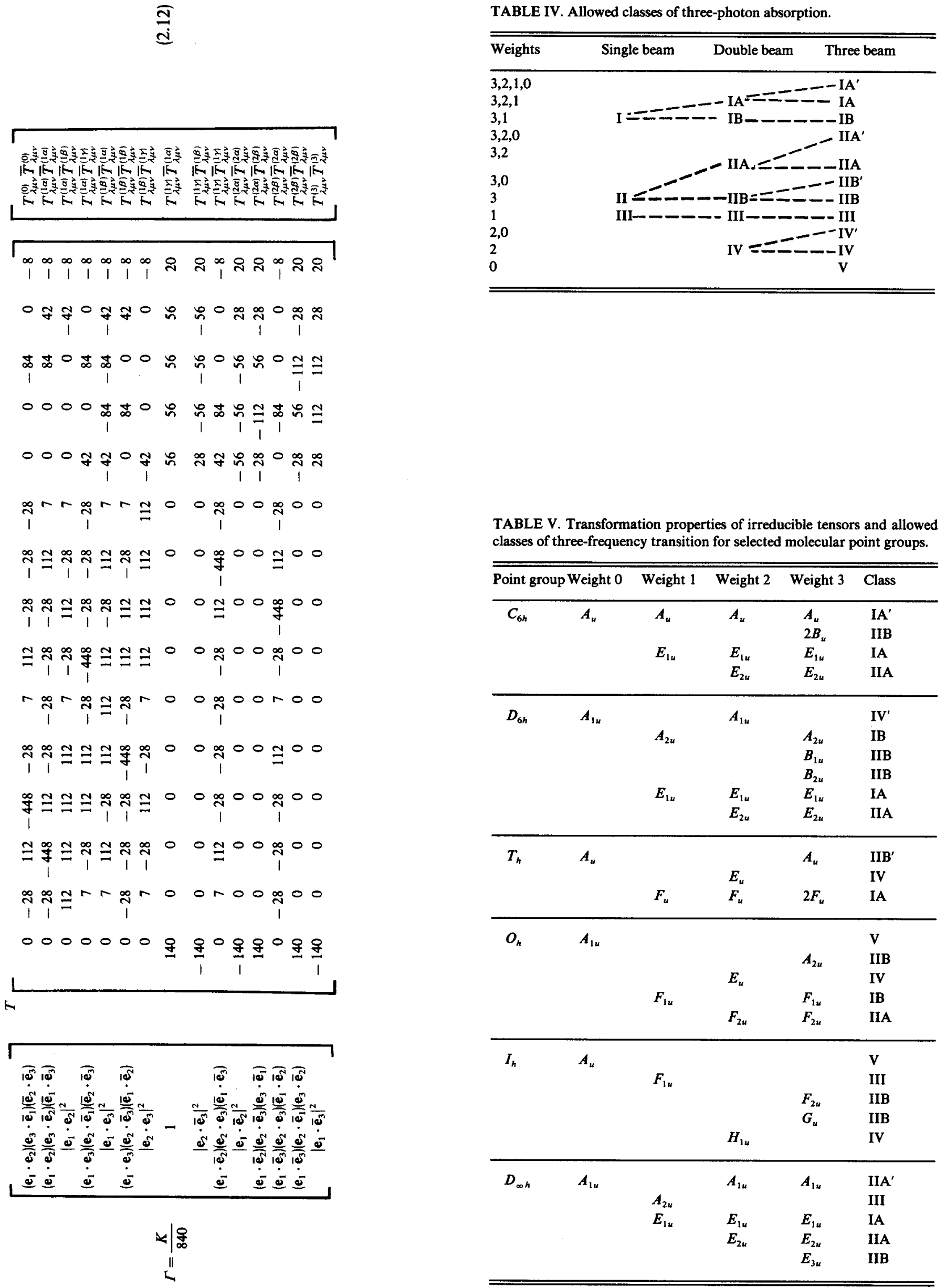


In the special case where the molecular response tensor may be regarded as real, i.e., when the excitation frequencies are far removed from resonance, the molecular parameters reduce down to a set of eleven linearly independent elements, and the following rate expression applies:

$$
\Gamma=\frac{K}{840}\left[\begin{array}{c} 
\\
\operatorname{Re}\left(\mathbf{e}_{1} \cdot \mathbf{e}_{2}\right)\left(\mathbf{e}_{3} \cdot \overline{\mathbf{e}}_{1}\right)\left(\overline{\mathbf{e}}_{2} \cdot \overline{\mathbf{e}}_{3}\right) \\
\operatorname{Re}\left(\mathbf{e}_{1} \cdot \mathbf{e}_{2}\right)\left(\mathbf{e}_{3} \cdot \overline{\mathbf{e}}_{2}\right)\left(\overline{\mathbf{e}}_{1} \cdot \overline{\mathbf{e}}_{3}\right) \\
\left|\mathbf{e}_{1} \cdot \mathbf{e}_{2}\right|^{2} \\
\operatorname{Re}\left(\mathbf{e}_{1} \cdot \mathbf{e}_{3}\right)\left(\mathbf{e}_{2} \cdot \overline{\mathbf{e}}_{1}\right)\left(\overline{\mathbf{e}}_{2} \cdot \overline{\mathbf{e}}_{3}\right) \\
\left|\mathbf{e}_{1} \cdot \mathbf{e}_{3}\right|^{2} \\
\left|\mathbf{e}_{2} \cdot \mathbf{e}_{3}\right|^{2} \\
1 \\
\left|\mathbf{e}_{2} \cdot \overline{\mathbf{e}}_{3}\right|^{2} \\
\left|\mathbf{e}_{1} \cdot \overline{\mathbf{e}}_{2}\right|^{2} \\
\operatorname{Re}\left(\mathbf{e}_{1} \cdot \overline{\mathbf{e}}_{2}\right)\left(\mathbf{e}_{2} \cdot \overline{\mathbf{e}}_{3}\right)\left(\mathbf{e}_{3} \cdot \overline{\mathbf{e}}_{1}\right) \\
\left|\mathbf{e}_{1} \cdot \overline{\mathbf{e}}_{3}\right|^{2}
\end{array}\right]
$$$$
\left[\begin{array}{r}
0 \\
0 \\
0 \\
0 \\
0 \\
0 \\
140 \\
-140 \\
-140 \\
280 \\
-140
\end{array}\right.
$$

$$
\begin{array}{rrr}
-56 & 168 & -952 \\
-56 & -952 & 168 \\
112 & 224 & 224 \\
14 & 168 & 168 \\
7 & 224 & -56 \\
7 & -56 & 224 \\
0 & 0 & 0 \\
0 & 0 & 0 \\
0 & 0 & 0 \\
0 & 0 & 0 \\
0 & 0 & 0
\end{array}
$$

$\begin{array}{rrr}14 & 168 & - \\ -56 & 168 & \\ 7 & -56 & \\ -56 & -952 & - \\ 112 & 224 & 7 \\ 7 & 224 & \\ 0 & 0 \\ 0 & 0 \\ 0 & 0 \\ 0 & 0 \\ 0 & 0\end{array}$

$\begin{array}{rrr}-56 & 0 & -168 \\ 14 & 0 & 168 \\ 7 & 0 & 0 \\ -56 & 84 & 168 \\ 7 & -42 & -168 \\ 112 & -42 & 0 \\ 0 & 56 & 112 \\ 0 & 28 & -112 \\ 0 & -56 & -112 \\ 0 & -56 & -112 \\ 0 & 28 & 224\end{array}$

Finally, a particular polarization configuration is worth noting. As shown in the Appendix, if the polarization vectors of the three beams are at a mutual angle of $\eta=62.9^{\circ}$, then all contributions to the spectrum other than those of weight 0 are suppressed. For this reason we shall refer to the arrangement as the weight 0 configuration, and we shall demonstrate its special significance in Sec. IV. For this configuration, the rate Eq. (2.12) simply reduces to

$$
\Gamma=K T_{\lambda \mu \nu}^{(0)} \bar{T}_{\lambda \mu \nu}^{(0)} \cos ^{3} \eta
$$

\section{SELECTION RULES}

In a multiphoton transition the symmetry of the final state of the molecule is characterized by a particular irreducible representation of the point group to which it belongs. For three-photon transitions originating from a totally symmetric ground state, the excited state symmetry dictates the representation under which components of the molecular response tensor must transform. Thus if a transition to a particular state is to be allowed, we require one or more of the irreducible tensors to transform under the irreducible representation of the final state. (If the molecular ground state is not totally symmetric, then it is the product of the initial and final state representations which determines the transformation properties.)

There are 11 distinct classes of transition, determined by the particular combination of irreducible weights allowed, as shown in Table IV. The classification scheme adopted here is a development of that used in earlier work. ${ }^{6,7}$ The three single-beam classes represent the only permitted transitions for the case where all three absorbed photons have the same frequency, and the six double-beam classes relate to the case where two photons have the same frequency, but the third differs. Clearly a much larger range of transitions can be induced if all three photons differ in frequency.

Table $\mathrm{V}$ presents an analysis of the classes of transition allowed by three-frequency absorption for six different highsymmetry molecular point groups. ${ }^{8}$ For example in a mole- cule of $\mathrm{O}_{h}$ symmetry such as sulphur hexafluoride $\left(\mathrm{SF}_{6}\right)$ weight 0 components of the transition tensor transform as $A_{1 u}$, weight 1 as $F_{1 u}$, weight 2 as $E_{u}+F_{2 u}$, and weight 3 as $A_{2 u}+F_{1 u}+F_{2 u}$. The table also shows that three-frequency transitions in such a molecule must belong to one of the five identifiable classes IB, IIA, IIB, IV, and V.

For molecules of lower symmetry, a classification of the allowed transitions is possible by using Table $\mathrm{V}$ in conjunction with correlation tables. ${ }^{8}$ However, caution must be exercised here; an example will illustrate the correct procedure. For a molecule of $C_{2 h}$ symmetry, such as trans-1, 2-dichloroethene, correlation tables for the descent in symmetry from $C_{6 h}$ to $C_{2 h}$ show that the representations map as $A_{u} \rightarrow A_{u} ; B_{u} \rightarrow B_{u} ; E_{1 u} \rightarrow 2 B_{u} ;$ and $E_{2 u} \rightarrow 2 A_{u}$. Using the results of Table $\mathrm{V}$, we thus find that for the group $C_{2 h}$, weight 0 transforms as $A_{u}$, weight 1 as $A_{u}+2 B_{u}$, weight 2 as $3 A_{u}+2 B_{u}$, and weight 3 as $3 A_{u}+4 B_{u}$. Hence, assuming a totally symmetric ground state, transitions to states $A_{u}$ and $B_{u}$ are allowed, the former belonging to class IA $^{\prime}$ (all weights allowed) and the latter to class IA (weights 1,2 , and 3 allowed). It is incorrect to directly deduce from the mapping $B_{u} \rightarrow B_{u}$ that transitions to states of this symmetry belong to the same class IIB in both point groups.

In passing it is worth noting that since the third rank molecular response tensor involves products of electric dipole transition moments, its transformation properties are determined from the relation:

$$
D^{1-} \otimes D^{1-} \otimes D^{1-}=D^{0-} \oplus 3 D^{1-} \oplus 2 D^{2-} \oplus D^{3-}
$$

and thus each weight is antisymmetric with respect to the inversion operator. Strictly, then, it is better to refer to weights $0^{-}, 1^{-}, 2^{-}$, and $3^{-}$, in order to provide a distinction from the tensor weights involved in two photon absorption, for example, which should be represented $0^{+}, 1^{+}$, and $2^{+}$.

\section{CONCLUSION}

In this paper, rate equations and selection rules have been provided for three-frequency absorption. By determin- 
ing the class to which such transitions belong, it is most often possible to provide unambiguous symmetry assignment for the excited state.

The procedure for determining the symmetry class can be made particularly simple. First, a double-beam experiment with the same three-photon frequency sum is carried out, and two polarization ratios are measured as described previously. ${ }^{7}$ This is sufficient to identify the class of all transitions allowed under the condition of double-beam excitation. Then, with three-frequency excitation in the special "weight 0 " configuration mentioned earlier, it is possible to determine whether any weight 0 contributions exist for these transitions. This completes the information required to assign each transition to one of the classes in the fourth column of Table V. Any completely new features in the weight 0 spectrum should correspond to class $V$ transitions, which have the distinction of being uniquely allowed by three-frequency excitation. Transitions of this class only arise in molecules belonging to one of the cubic point groups; an example is afforded by a transition of $A_{2}$ symmetry in a tetrahedral molecule belonging to the group $T_{d}$.

\section{APPENDIX: DERIVATION OF THE CONDITIONS FOR THE WEIGHT O CONFIGURATION}

The rate Eq. (2.1) may be expressed as

$$
\Gamma=K\left\langle\left|T_{i j k} S_{i j k}\right|^{2}\right\rangle \text {, }
$$

where

$$
S_{i j k}=e_{1 i} e_{2 j} e_{3 k} .
$$

Under conditions where the polarization tensor $S_{i j k}$ is of purely weight 0 character, then

$$
S_{i j k}=S_{i j k}^{(0)}
$$

and $(\mathrm{A} 1)$ reduces to

$$
\Gamma=K\left\langle\left|T_{i j k}^{(0)} S_{i j k}^{(0)}\right|^{2}\right\rangle
$$

hence only weight 0 contributions to the molecular tensor appear in the rate equation.

From Eq. (2.8) of the main text, (A3) may be written as

$$
e_{1 i} e_{2 j} e_{3 k}=\frac{1}{6} \epsilon_{i j k}\left(\mathbf{e}_{1} \times \mathbf{e}_{2}\right) \cdot \mathbf{e}_{3}
$$

and contraction with $e_{2 i} e_{3 j} e_{1 k}$ results in the equation

$$
\left(e_{1} \cdot e_{2}\right)\left(e_{2} \cdot e_{3}\right)\left(e_{3} \cdot e_{1}\right)=\frac{1}{6}\left[\left(e_{1} \times e_{2}\right) \cdot e_{3}\right]^{2} \text {. }
$$

For this relation, it is readily shown by simple vector algebra that if we have three plane polarized beams intersecting in such a way that the angle between each pair of polarization vectors is the same, i.e.,

$$
\left(e_{1} \cdot e_{2}\right)=\left(e_{2} \cdot e_{3}\right)=\left(e_{3} \cdot e_{1}\right)=\cos \eta
$$

then we have

$$
\cos ^{3} \eta=\frac{1}{6}\left(1-3 \cos ^{2} \eta+2 \cos ^{3} \eta\right) \text {. }
$$

Hence $\cos \eta$ is the real root of the cubic equation

$$
1-3 \cos ^{2} \eta-4 \cos ^{3} \eta=0
$$

which leads to the result $\eta=62.909^{\circ}$.

'D. L. Andrews and T. Thirunamachandran, J. Chem. Phys. 67, 5026 (1977).

${ }^{2}$ W. M. McClain, J. Chem. Phys. 57, 2264 (1972).

${ }^{3}$ D. L. Andrews and T. Thirunamachandran, J. Chem. Phys. 68, 2941 (1978).

${ }^{4}$ In earlier work (Refs. 5-7), products of weight 1 tensors have been expressed with an alternative index contraction such as $T_{\lambda+\psi}^{(i n} \bar{T}_{\lambda w}^{(i)}$. However, it is readily shown that such products are related to those of the form used in this paper by a simple numerical factor, for example $T_{\lambda \mu \mu}^{(1 \eta p} \bar{T}_{\lambda \nu}^{(1 \gamma)}$ $={ }_{2} T_{\lambda \mu \nu}^{(\lambda \eta t} \bar{T}_{\lambda \mu \nu}^{(1) i}$.

${ }^{5}$ D. L. Andrews and W. A. Ghoul, J.Chem. Phys. 75, 530 (1981).

${ }^{6}$ D. L. Andrews, J. Chem. Phys. 77, 2831 (1982).

${ }^{7}$ D. L. Andrews, J. Chem. Phys. 78, 1731 (1983)

${ }^{8}$ The symmetry states referred to in Table V are as given in J. A. Salthouse and M. J. Ware, Point Group Character Tables and Related Data (Cambridge University, Cambridge, 1972). 